\title{
Displacement ductility demand and strength reduction factors for rocking structures
}

\author{
M. Trueb ${ }^{1}$, Y. Belmouden ${ }^{2} \&$ P. Lestuzzi ${ }^{2}$ \\ ${ }^{1}$ ETHZ-Swiss Federal Institute of Technology Zurich, Switzerland \\ ${ }^{2}$ EPFL-Ecole Polytechnique Fédérale de Lausanne, Switzerland
}

\begin{abstract}
This paper reports the main results of an extensive parametric study using numerical simulations and computing displacement ductility demand of nonlinear single-degree of freedom (SDOF) systems and multi-degree of freedom (MDOF) systems for a set of 164 registered ground motions. The objective of this study is to propose values of strength reduction factors for rocking behavior for seismic analysis. In the first part focused on SDOF systems, non-linear seismic responses obtained with a hysteretic model simulating rocking are statistically compared with the ones related to established hysteretic models for ductile structures. Similar to established hysteretic models, results confirm that the frequency has little influence on the ductility demand if it is below $2 \mathrm{~Hz}$ and a substantial influence if it is above $2 \mathrm{~Hz}$. Moreover, they show that the other parameters, especially the hysteretic behavior model, have only little influence on the displacement ductility demand. Surprisingly, displacement ductility demand is found to be practically independent of the additional viscous damping ratio. Finally, a relationship between displacement ductility demand and strength reduction factor for rocking systems is proposed. The second part shows that the results obtained for SDOF systems are also valid for MDOF systems.

Keywords: displacement ductility demand, strength reduction factor, non-linear structural response, rocking, earthquake, seismic analysis, hysteretic model.
\end{abstract}

\section{Introduction}

Intensive numerical investigations have already been performed to examine the relationships between strength reduction factors and non-linear behavior of structures subjected to earthquake ground motions (see [1] for a review of 
significant investigations). The studies were generally focused on non-linear single-degree-of-freedom (SDOF) systems defined by different hysteretic models. However, the involved hysteretic models (elastoplastic, Clough, Takeda, etc.) were mostly related to seismic behavior with significant energy dissipation such as ductile reinforced concrete shear walls. Until recently, no systematic investigations were carried out for structures without hysteretic energy dissipation capacity such as slender unreinforced masonry shear walls that show very different seismic behavior. Other structures that show this type of behavior are precast post-tensioned reinforced concrete structures or concentrically braced steel structures with slender diagonal elements.

This paper presents the main results gained during the master thesis performed by the first author at the Swiss Federal Institute of Technology in Lausanne (EPFL). More complete description of this work may be found in [2]. The research project aims to answer the following question: under what conditions can the strength reduction factor for structures without hysteretic energy dissipation capacity be extended beyond the limited value of 1.5 accounting for overstrength only proposed by the construction codes?

\section{Methodology}

The methodology used in this study consists first of a systematic investigation of the non-linear response of SDOF systems subjected to a set of 164 earthquake recordings. Figure 1 illustrates the methodology schematically. The structural behavior is described by a hysteretic model developed for simulating non-linear behavior without hysteretic energy dissipation capacity and by two recognised hysteretic models as reference.

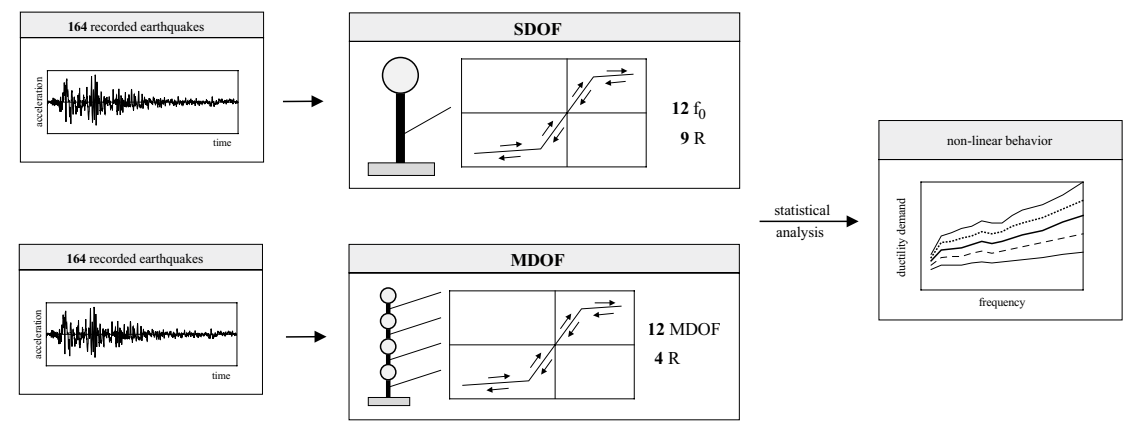

Figure 1: Schematic description of the followed methodology.

Statistical analysis of the seismic response is performed for twelve initial natural frequencies $\left(\mathrm{f}_{0}\right)$ representing the typical range of natural frequencies of buildings and for nine values of the strength reduction factor (R). The displacement ductility demand is considered to be a representative indicator for the non-linear seismic behavior. The investigations are later extended to MDOF systems. The motivation behind this second part of the investigations is to test if 
the results obtained for SDOF systems hold true for MDOF systems representing buildings.

\section{Ground motions}

164 registered ground acceleration time histories are used. In order to consider earthquakes that may produce significant non-linearities in the structural behavior, only recordings with a magnitude larger than 5 were considered. Figure 2 shows the magnitude-epicentral distance relationship of the set of 164 selected recordings. The magnitudes range from 5.0 to 7.6, the epicentral distances range from 2 to $195 \mathrm{~km}$ and the peak ground accelerations (PGA) range from 0.61 to $7.85 \mathrm{~m} / \mathrm{s}^{2}$.

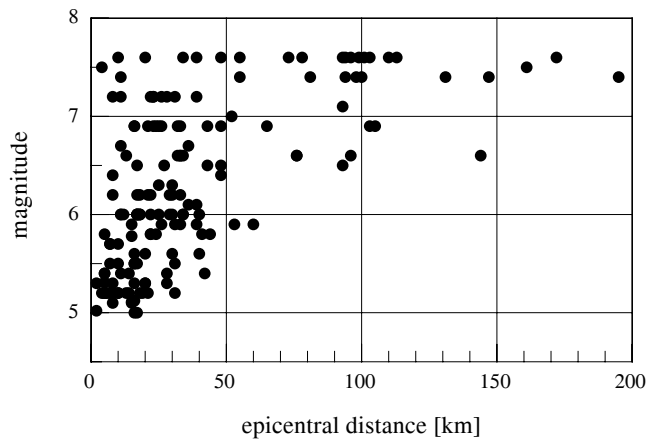

Figure 2: $\quad$ Magnitude-epicentral distance relationship of the 164 recordings.

\section{Investigations with SDOF systems}

According to the methodology illustrated in Figure 1, the following parameters are examined in the first part of the study with SDOF systems: the initial natural frequency, the strength reduction factor, the hysteretic energy dissipation capacity using three hysteretic models and the viscous damping ratio. The nonlinear SDOF system is defined by the following parameters: the initial natural frequency $\left(\mathrm{f}_{0}\right)$, the strength reduction factor $(\mathrm{R})$ and the hysteretic model.

Twelve initial natural frequencies covering the range of frequencies of usual buildings are evaluated. The natural frequencies range from $\mathrm{f}_{0}=0.5 \mathrm{~Hz}$ to $4.0 \mathrm{~Hz}$ in steps of $0.25 \mathrm{~Hz}$. The following hysteretic models are used to compute the non-linear responses: a bilinear self-centring model (S-model), an elastoplasticmodel and the modified Takeda-model. The force-displacement relationships defining the S-model and the modified Takeda model are plotted in Figure 3.

The bilinear self-centring hysteretic model is the simplest model to represent elements without or very little hysteretic energy dissipation capacity. It is called self-centring because it unloads such that there is no residual displacements when the external load is reduced to zero. Because of its shape, this model is 
called "S-model". The post-yield stiffness is defined as being a fraction of the initial stiffness.

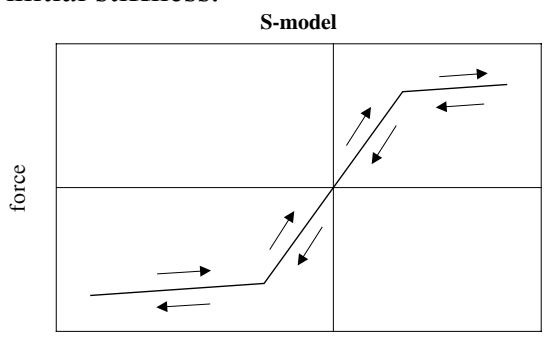

displacement

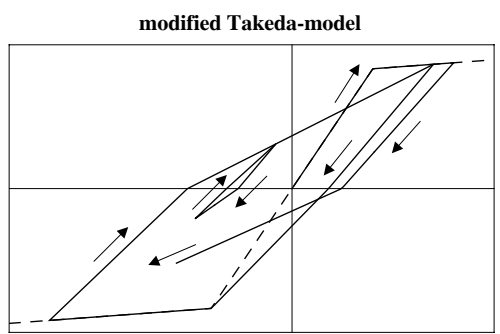

displacement

Figure 3: The S-model and the modified Takeda model.

The modified Takeda-model simulates well the features of ductile structures such as capacity designed reinforced concrete structures. The Takeda-model was initially proposed by Takeda et al. [3]. It was later modified by many researchers. The version used here is the one of Allahabadi and Powell [4].

\subsection{Results with SDOF systems}

Relative displacements are used to represent the dynamic non-linear response. Because the computations are repeated for each recording, 164 values are used to determine the average and standard deviation for each couple of strength reduction factor and initial fundamental frequency.

The results for the displacement ductility demand are presented first, in terms of mean values and in terms of variability. Later section relates the impact of the damping ratio on the non-linear behavior.
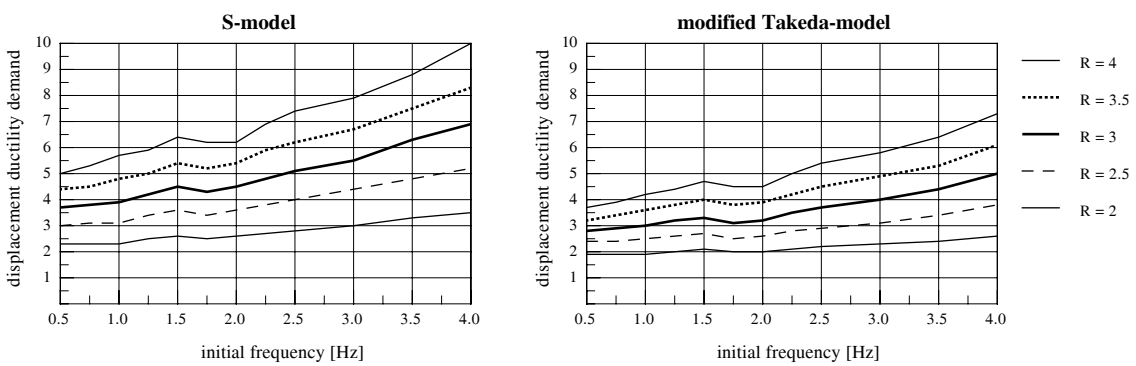

Figure 4: Mean values of the displacement ductility demand as a function of the initial frequency of SDOF system.

\subsubsection{Mean values of displacement ductility demand}

The displacement ductility demand $\left(\mu_{\Delta}\right)$ is defined as the ratio of the peak nonlinear displacement to the yield displacement. The displacement ductility demand varies strongly between different considered ground motions but mean values obtained from a large number of ground motions show clear tendencies. 
Typical results are illustrated in Figure 4. The plotted results correspond to a post-yield stiffness equal to $10 \%$ of the initial stiffness for both hysteretic models. The plots show very similar tendencies. As expected, larger displacement ductility demands are related to S-model. However, the differences are not pronounced. Moreover, the general shape of the curves is conserved. The displacement ductility demand stays more or less constant for frequencies below $2 \mathrm{~Hz}$ and afterwards increases with increasing frequency.

\subsubsection{Variability of displacement ductility demand}

Besides mean values, variability is the main statistical characteristic of the displacement ductility demand. Typical results are illustrated in Figure 5 for one value of the strength reduction factor $(R=3)$. In order to characterize the variability, the mean values (solid line) are plotted together with mean values plus one standard deviation and mean values minus one standard deviation (dotted lines) as a function of the initial frequency of the SDOF systems. Based on the plots of Figure 5, the comparison between the S-model and the modified Takeda-model shows that even if variability is significantly larger for the S-model, there are similarities in both hysteretic models. Variability stays approximately constant for frequencies below $2 \mathrm{~Hz}$ and significantly increases afterwards.
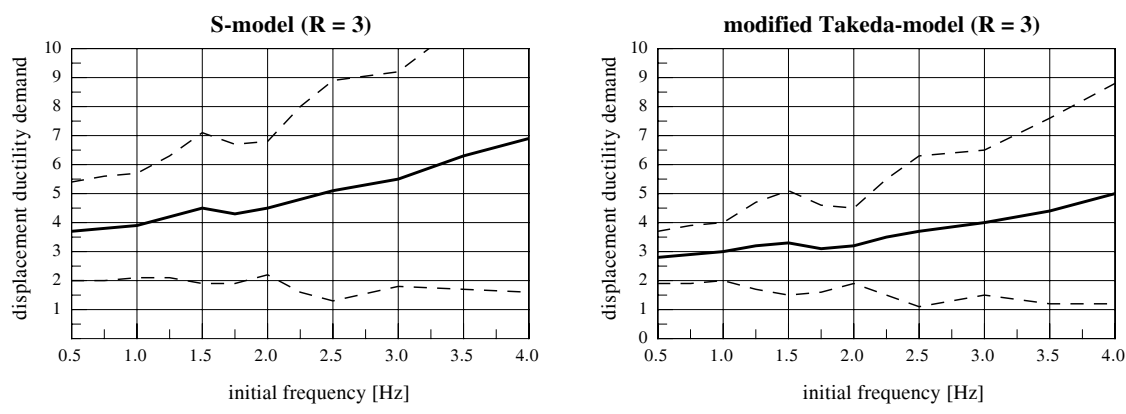

Figure 5: Variability of the displacement ductility demand as a function of the initial frequency of the SDOF system.

\subsubsection{Viscous damping ratio}

For the viscous damping ratio, the performed parametric study generated unexpected results. Figure 6 shows typical results. The displacement ductility demand stays approximately constant for all considered damping ratios except those between $0 \%$ and $1 \%$. The displacement ductility demand is smaller than the obtained plateau for damping ratios between $0 \%$ and $1 \%$ and gradually increases in this range until it stabilises at a constant value. This phenomenon is independent of the initial frequency and the value of strength reduction factor. Obviously, the damping ratio reduces the elastic and the non-linear response by the same amount. 


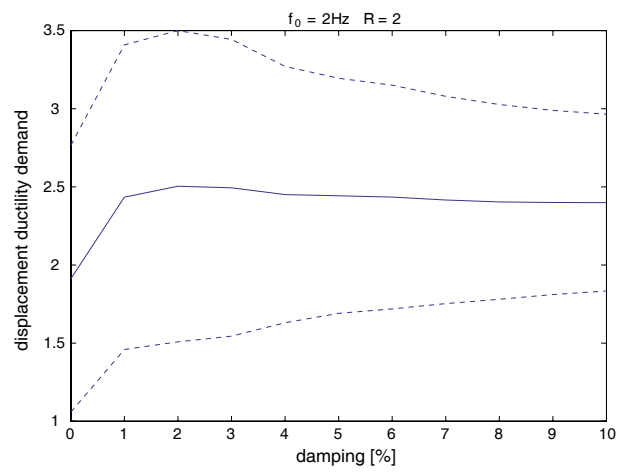

Figure 6: The impact of the viscous damping ratio is restricted to the range between $0 \%$ and $1 \%$.

\subsection{A simplified formulation for $R-\mu_{\Delta}-T$ relationship}

The main objective of the research project is to propose strength reduction factor-displacement ductility demand relationship for structures without hysteretic energy dissipation capacity. However, similar to the equal displacement rule, the formulation should remain as simple as possible. In brief, for structures without capacity of hysteretic energy dissipation, the study is focused on the improvement of the equal displacement rule for the frequency range below $2 \mathrm{~Hz}$, particularly for strength reduction factors between 2 and 3 . Figure 4 shows that the equal displacement rule $\left(\mu_{\Delta}=\mathrm{R}\right)$ leads to underestimating the results for all frequencies above $0.5 \mathrm{~Hz}$. By contrast, the usual competing empirical rule of equal energy $\left(\mu_{\Delta}=R^{2} / 2+1 / 2\right)$ leads to largely overestimated results for strength reduction factors above $\mathrm{R}=2$ (e.g. $\mu_{\Delta}=5$ for $\mathrm{R}=3$ ). Consequently convenient relationship should lies between these two common empirical rules. As a boundary condition, the relationship should lead to $\mu_{\Delta}=1$ for $\mathrm{R}=1$. Based on the results of the parametric study, a simplified formulation for $\mathrm{R}-\mu_{\Delta}-\mathrm{T}$ relationships is proposed as follows:

$$
\mu_{\Delta}=3 \mathrm{R} / 2-1 / 2 ; \quad \mathrm{T}>0.5 \mathrm{~s} .
$$

The proposed $\mathrm{R}-\mu_{\Delta}$ relationship is printed in Figure 7 and plotted together with the obtained results of Figure 4. The relationship (1) is set to be valid in terms of mean values for the frequency range below $2 \mathrm{~Hz}$ and for strength reduction factors between 2 and 3. The relationship should be adjusted if it is to be used for higher strength reduction factors. One suggested modification consists of removing the constant member. Note that for $\mathrm{R}=2$, Equation (1) and the empirical equal energy rule lead to identical results $\left(\mu_{\Delta}=2.5\right)$. 

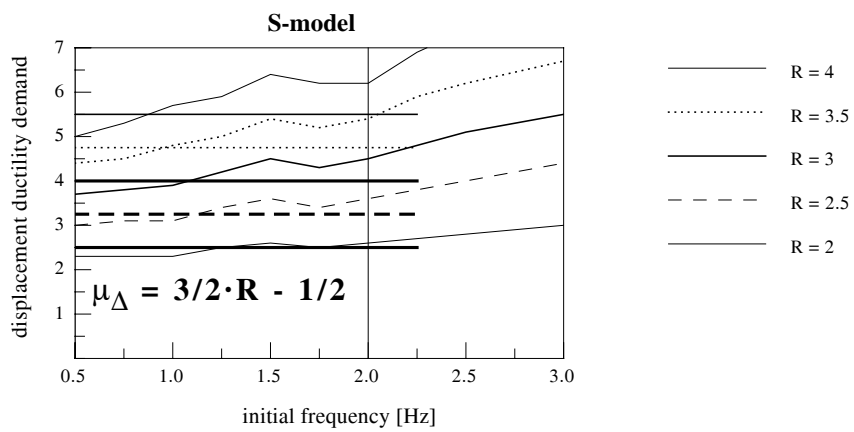

Figure 7: $\quad$ Proposed $\mathrm{R}-\mu_{\Delta}$ relationship in comparison with the results.

\section{Investigations with MDOF systems}

In order to verify the validity of the results obtained for SDOF systems for multistorey structural wall buildings, a second investigation is performed with MDOF systems. Non-linear responses are computed using the same database of 164 recordings. The same type of non-linear constitutive law according to the S-model is used for every storey of the MDOF system.

\subsection{Definition of MDOF systems}

Figure 8 shows an example of the structures which were used in this part of the study. The model represents a building with four stories. The mass of the building is modelled as a concentrated mass (M) at each story level and it is kept the same for every story. The slabs are considered infinitely rigid in their inplane direction and no rotational degrees of freedom are introduced. Each story has one horizontal lateral displacement degree of freedom. All the stories are modelled with the S-model. This hypothesis is based on the assumption that the slabs are infinitely rigid and therefore every wall element between the slabs can undergo a rocking behavior with no coupling effect. All other failure mechanisms, such as sliding or shear, are excluded.
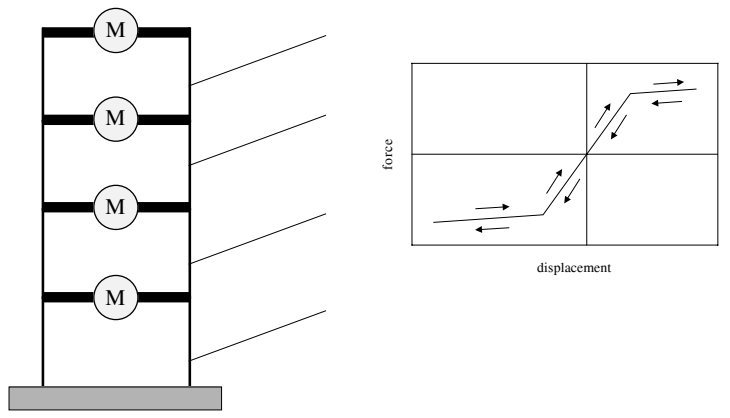

Figure 8: Sketch for a four-story structure used in the MDOF systems investigations. 
The determination of the displacement ductility demand is carried out for a two story, a four story and a six storey building model. A parametric study is performed for four values of the initial story stiffness $(K=100 \mathrm{~N} / \mathrm{m}, 500 \mathrm{~N} / \mathrm{m}$, $1000 \mathrm{~N} / \mathrm{m}$ and $2000 \mathrm{~N} / \mathrm{m}$ ) and for four values of the strength reduction factor $(\mathrm{R}=1.5,2.0,3.0$ and 4.0$)$. The total mass is equal to unity. The resulting fundamental frequency for all MDOF systems investigated is given in Table 1 .

Table 1: Fundamental frequencies of the MDOF systems.

\begin{tabular}{|c|c|c|c|}
\hline \multirow{2}{*}{$\begin{array}{c}\text { Initial stiffness } \\
{[\mathrm{N} / \mathrm{m}]}\end{array}$} & \multicolumn{3}{|c|}{ Frequency [Hz] } \\
\cline { 2 - 4 } & $2 \mathrm{DOF}$ & 4 DOF & 6 DOF \\
\hline 100 & 1.4 & 1.1 & 0.9 \\
\hline 500 & 3.1 & 2.5 & 2.1 \\
\hline 1000 & 4.4 & 3.5 & 3.0 \\
\hline 2000 & 6.2 & 5.0 & 4.2 \\
\hline
\end{tabular}

\subsection{Equivalent SDOF system}

To ensure a relevant comparison of the results between MDOF and SDOF systems, equivalent SDOF systems are defined for each MDOF system. An equivalent SDOF system follows the same hysteretic model as the stories of the corresponding MDOF system (S-model). Thus both systems have the same initial fundamental frequency. However, the post-yield stiffness for the equivalent SDOF system should be calibrated to reproduce the same global behavior as the corresponding MDOF system. The equivalence is determined on the basis of push-over curves and leads to a modification (multiplication) of the hardening coefficient for equivalent SDOF systems (1.2 times for 2 DOF, 0.8 times for $4 \mathrm{DOF}$ and 0.7 times for $6 \mathrm{DOF}$ systems).

\subsection{Displacement ductility demand}

The computation of displacement ductility demand with MDOF systems is not as straightforward as with SDOF systems. It is important to distinguish between local and global ductility. The $\mathrm{R}-\mu_{\Delta} \mathrm{T}$ relationships are expressed for global displacement ductility demands. For example, the equal displacement rule is formulated for the global displacement ductility demand of a structure. Therefore, the comparison of the displacement ductility demand between SDOF and MDOF systems needs to be done of the basis of the global displacement ductility demand. The global displacement ductility demand is defined as the peak non-linear displacement at the top of the building divided by the top displacement at the stage when the first element reaches its yield relative displacement. The global yield displacement is the peak linear elastic displacement of the top of the building divided by the corresponding strength reduction factor. 


\subsection{Results with MDOF systems}

The displacement ductility demand is chosen as a representative value for the non-linear behavior. In virtue of the discussion above, the global ductility is used to compute the displacement ductility demand. The results are plotted in Figure 9 as a function of the fundamental frequencies of the examined structures.
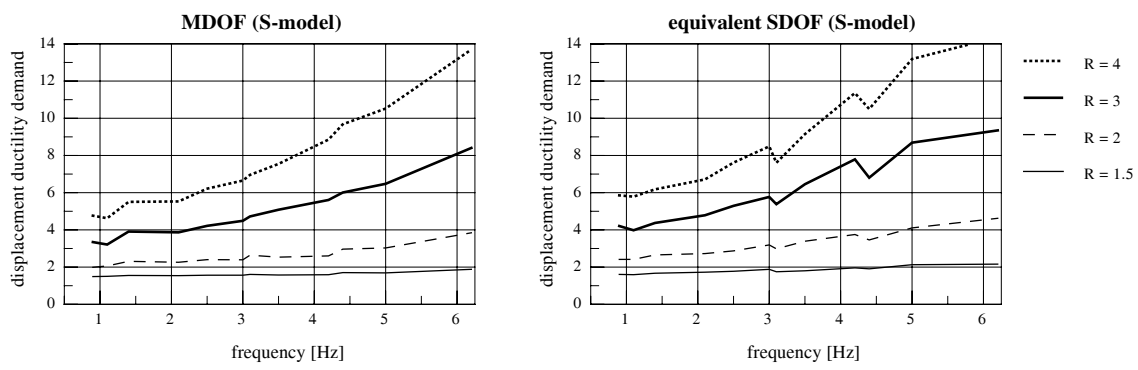

Figure 9: Mean values of the displacement ductility demands for MDOF systems and related equivalent SDOF systems.

The plots of Figure 9 show that the equivalent SDOF system (right) generally overestimates the displacement ductility demand when compared to the corresponding MDOF system (left). The difference lies between $10 \%$ and $15 \%$. In the adopted methodology, some equivalent SDOF systems have a similar initial natural frequency (see Table 1) but a quite different post-yield stiffness ratio. This explains the abrupt drops in the force-displacement curves of the equivalent SDOF systems (Figure 9, right).

\section{Summary and conclusions}

In this paper, the seismic response of structures that show a non-linear rocking behavior such as slender unreinforced masonry shear walls or precast posttensioned reinforced concrete elements is investigated. The displacement ductility demand is computed for a set of 164 registered ground motions. Statistical analyses are performed to characterize seismic performance. The obtained results reveal that hysteretic models without hysteretic energy dissipation capacity definitely do not lead to excessive displacement ductility demand. This is an important result that contradicts the widely held perception. It is often assumed that this kind of structural behavior is not an efficient mechanism to withstand strong earthquakes, even if it may be associated with significant deformation capacity. In the light of the presented results it is found that hysteretic energy dissipation capacity is not the unique characteristic of a good seismic behavior. The non-linear behavior due to the transition between initial stiffness and post-yield stiffness is the main favourable aspect that affects seismic behavior. 
Note that since different yield displacements are considered for the definition of the non-linear systems, the results obtained for the displacement ductility demand may not be extended to those for the displacement demand.

Compared to non-linear SDOF systems, similar seismic behavior is also seen in MDOF systems. However, the SDOF system has a tendency to overestimate the displacement ductility demand of the corresponding MDOF system by about $15 \%$.

The upper-limit value of 1.5 , currently being recommended by the design codes for strength reduction factors of structures with limited hysteretic energy dissipation capacity considering only their overstrength is definitely too conservative. As long as the structural elements have a large displacement capacity, strength reduction factors up to 3 can be adopted. Note that additional attention should be paid to the fact that no other structural failure mechanism can take place and that strength degradation may be excluded. For frequencies below $2 \mathrm{~Hz}$ a prediction of the displacement ductility demand may be obtained by using the proposed $\mathrm{R}-\mu_{\Delta}-\mathrm{T}$ relationships. This conclusion is important for many cases. One example are slender unreinforced masonry elements subjected exclusively to the "rocking" failure mode.

\section{References}

[1] Miranda E. and Bertero V., Evaluation of Strength Reduction Factors for Earthquake-Resistant Design. Earthquake Spectra. Vol 10, No. 2, pp. 357379, 1994.

[2] Trueb M., Seismic behaviour of non-linear elements with little energy dissipation. Master thesis, Ecole Polytechnique Fédérale de Lausanne (EPFL), Switzerland, 2005.

[3] Takeda T., Sozen M. A. and Nielsen N. N., Reinforced concrete response to simulated earthquakes. Journal of the Structural Division. Proceedings of the American Society of Civil Engineers (ASCE). Vol. 96, No. ST12, 1970.

[4] Allahabadi R. and Powell G. H., Drain-2DX User Guide. Report No. UCB/EERC-88/06. College of Engineering, University of California, Berkeley, 1988. 